\title{
Caracterización de la intensidad de señal de la glándula mamaria mediante imagen por resonancia magnética en modelo animal canino
}

\author{
Characterization of mammary gland signal intensity by \\ magnetic resonance imaging
}

\author{
Xavier Leonardo Jaramillo-Chaustre \\ Ph.D. Medicina y Cirugía Animal \\ Universidad de Pamplona \\ Pamplona, Colombia \\ xavier.jaramillo@unipamplona.edu.co
}

\author{
Alicia Ester Serantes-Gómez \\ Ph.D. Medicina y Cirugía Animal \\ Universidad de León \\ León, España \\ aeserg@unileon.es
}

Jhon Jairo Bustamante-Cano
Ph.D. Medicina y Cirugía Animal
Universidad de Pamplona
Pamplona, Colombia
jhonjabu@unipamplona.edu.co quently developed, addition to become acquainted with the software used. To validate the technique used in the images they were considered three structures (air, fat and muscle) which IS showed significant difference between glandular breast tissue (IS $184.8 \pm 28.2$ ) and regional lymph nodes (IS $51.3 \pm 5,4$ ), allowing to determine the benchmark for the study. It is concluded that the use of a low field equipment of MRI provides sufficient information for the mammary gland, ducts and regional lymph nodes study in dogs, making a complete anatomical evaluation and identification of such structures.

Keywords: Breast, dogs, magnetic resonance image, signal strength.

\section{INTRODUCCIÓN}

La glándula mamaria es el órgano distintivo de los mamíferos y consiste en una estructura glandular especializada en la síntesis y secreción de leche [1], [2], [3]. La perra dispone, por lo general, de cinco complejos mamarios dispuestos a ambos lados del surco intermamario, que se denominan torácico craneal, torácico caudal, abdominal craneal, abdominal caudal e inguinal. Cada complejo mamario consta de un cuerpo mamario que contiene de dieciséis a veinte glándulas mamarias y su correspondiente orificio de senos lactíferos, así como de una papila mamaria con sus conductos y agujeros papilares. Los senos 
lactíferos (colectores de leche) están divididos en una parte glandular, que corresponde al cuerpo, y una parte papilar.

En cuanto a los vasos sanguíneos del sistema craneal, parten de la arteria torácica lateral para la mama torácica craneal y de la arteria torácica interna, estas, junto con sus venas satélites, discurren con las ramas perforantes de la arteria torácica interna cerca de la línea alba para irrigar las dos glándulas mamarias torácicas y posteriormente continuar como la arteria y vena epigástricas superficiales que, a la altura del arco costal, atraviesan la pared torácica y, después de irrigar las mamas abdominales craneales a nivel del ombligo se anastomosan con los vasos caudales del mismo nombre. Los vasos sanguíneos del área de abastecimiento caudal se originan en la arteria pudenda externa que se divide, después de atravesar el espacio inguinal a la altura de la mama inguinal, en las ramas labiales ventrales o escrotales ventrales y en la arteria epigástrica caudal superficial, que a su vez dan las ramas mamarias o prepuciales correspondientes [2], [3], [4], [5], [6], [7].

La inervación de la glándula mamaria está constituida por un componente sensitivo (fibras sensitivas somáticas) que recibe de la superficie cutánea la sensación táctil y térmica generando los estímulos externos que envía luego, por vía aferente, al sistema nervioso central, y un componente simpático motor que forma parte del sistema nervioso autónomo. El componente simpático inerva las células mioepiteliales de toda la cisterna, y las fibras sensitivas están presentes a nivel del pezón [8].

Rendimiento e interpretación del tejido mamario en IRM. El tejido mamario es muy sensible a cambios hormonales, lo que da lugar a cambios morfológicos en todo el ciclo reproductivo. La mayoría de los estudios muestran, en humanos, que las primeras fases del ciclo menstrual, los días 3 al 14 (etapas de proliferación folicular), son los mejores para realizar un examen mediante IRM, ya que reducen los casos espontáneos de realce benigno dada la alta sensibilidad de esta herramienta de diagnóstico por imagen en el estudio de esta zona anatómica, ya que aporta no solo una imagen estructural sino funcional de la estructura en estudio y como si fuese poco solo con el manejo del software del equipo se puede mejo- rar considerablemente la región para estudiar [9], [10]. También se debe tener en cuenta el historial de tratamientos hechos en la mama, como terapia hormonal de reemplazo y biopsias para emitir un diagnóstico sobre el posible problema [11].

\section{MATERIALES Y MÉTODOS}

Para la realización del presente estudio se utilizaron un total de 12 individuos de la especie canina, todos ellos hembras, que no presentaban alteraciones patológicas en la glándula mamaria. Con edades comprendidas entre 1 y 11 años y un peso comprendido entre 5,8 y 65,0 kg. Todas ellas habían sido remitidas a la Unidad de Cirugía y Diagnóstico por Imagen (UCDI) de la Facultad de Veterinaria de la Universidad de León (FVULE) por otros centros veterinarios, o bien admitidas directamente en nuestro servicio, por motivos absolutamente ajenos a problemas mamarios, reproductivos o endocrinos. Se les realizó una exploración exhaustiva de las mamas, incluyendo el estudio imaginológico mediante resonancia magnética, siempre previo consentimiento expreso de sus propietarios.

\section{1 Material fungible empleado en la anestesia}

En el protocolo anestésico utilizado para la realización de los estudios mediante IRM se administraron soluciones de Midazolam y Buprenorfina, como preanestésicos. Propofol en solución como agente inductor de la anestesia, elegido por su efecto ultracorto y su rápida metabolización y eliminación intra y extrahepática, e isofluorano para el mantenimiento de la anestesia general, así como cal sodada para la absorción de $\mathrm{CO}_{2}$ durante este periodo.

Para la aplicación, fijación y suministro de fármacos anestésicos y fluidos se emplearon catéteres de teflón de calibre 24, 22 y 20 G, además de compresores de caucho para facilitar la visualización de la vena, jeringuillas de distintos tamaños, agujas de diferentes diámetros y longitudes, sistemas de suero y suero salino fisiológico, así como sondas endotraqueales con manguito de neumotaponamiento de diferentes calibres para adaptarse a los diámetros traqueales correspondientes a perros de 6 a $65 \mathrm{Kg}$ de peso, siguiendo la técnica rutinaria descrita en la bibliografía [12], [13], [14]. Toda la información se refleja en la ficha anestésica. 
Para la anestesia general de los animales durante la exploración, mediante IRM, se utilizó un aparato de anestesia inhalatoria marca MDS MATRIX® (modelo VML), especialmente diseñado para su uso en instalaciones de RM (no es posible la utilización de un equipo de anestesia convencional, debido a que sus componentes ferromagnéticos se verían afectados por la presencia del imán permanente) con un vaporizador para isofluorano de la marca DRAGER ${ }^{\circledR}$. El equipo se conecta directamente al circuito general de gases del Servicio de Cirugía de la FVULE.

\subsection{Exploración mediante IRM}

Una vez anestesiados, los pacientes se colocaron en la camilla móvil del equipo de RM, con ayuda de diversos posicionadores específicos para el modelo y equipo de RM de que disponíamos, en decúbito supino, con la cabeza en dirección distal con respecto al imán, las extremidades torácicas extendidas en dirección craneal, las pelvianas en dirección caudal y la región por estudiar lo más centrada posible dentro de este. A continuación, se eligió, en función de la talla del paciente, una antena de radiofrecuencia que fue colocada alrededor de la zona que comprende la glándula mamaria inguinal, de tal forma que en las imágenes obtenidas se pudieran visualizar tanto la mama como los conductos linfáticos que drenan la linfa hacia los ganglios inguinales. Las antenas utilizadas fueron: la de "cuerpo" para los animales de 55 a $65 \mathrm{~kg}$, la de cabeza para los animales de talla media, de 21 a 31kg, y la del tipo 6 pulgadas para los de peso inferior a $20 \mathrm{~kg}$, como describen Gili [17], Millán [12], Godoy [13] y Duarte [14].

Para la realización de los exámenes mediante IRM se emplearon las instalaciones específicas aisladas mediante cobre en la UCDI, en cuyo interior se encuentra el equipo de RM (GENERAL ELECTRIC MEDICAL SYSTEMS ${ }^{\circledR}$ ) modelo SIGNA PROFILE 2, con un imán permanente de 0,2 Teslas.

Para el estudio de la glándula mamaria en los pacientes durante el protocolo de exploración, la primera serie que se programó fue el "localizador multiplanar" o de tres planos, serie potenciada en T1 que se obtiene en el plano sagital y cuya función no es otra que encontrar la región anatómica del animal que se va a explorar en el interior del campo magnético. Para la adquisición de estas imágenes se utilizó un TR de 51 ms, un TE de 18 $\mathrm{ms}$, con un espesor de corte de $6 \mathrm{~mm}$ y una separación entre ellos de $2 \mathrm{~mm}$; se utilizó una matriz de adquisición de 256 por 192, que fue empleada en todos los casos del estudio.

Posteriormente, basándonos en el localizador multiplanar, se programaron las distintas series orientándolas manualmente sobre este, prestando especial atención al hecho de que los cortes programados fueran numéricamente suficientes como para cubrir toda la región de interés, así como para que el sentido del corte fuera lo más correcto posible; en nuestro caso, el ángulo empleado fue de $70^{\circ}$, mostrándonos con especial detalle las estructuras de interés.

Tras obtener el localizador, se programó una serie potenciada en T1, por aportar una mejor calidad de imagen anatómica de la región para estudiar, tanto de la glándula mamaria como de otras estructuras de la región. Con esta serie recopilamos información para su posterior estudio y análisis; en nuestro caso, se emplearon valores de TE que oscilaron entre 32 y $34 \mathrm{~ms}$, mientras que los valores de TR variaron entre 520 y 540 $\mathrm{ms}$, el espacio entre cortes entre 4,5 y $5,5 \mathrm{~mm}$ y el grosor de los cortes entre 5 y $7 \mathrm{~mm}$.

\subsection{Metodología y validación de la técnica empleada}

Para la validación de la técnica y la metodología empleadas, antes de iniciar las valoraciones, efectuamos una serie de mediciones en tres estructuras diferentes, para observar la variación en la escala de grises en una misma estructura y tomarla como referencia posteriormente, además de familiarizarnos de este modo con el programa informático empleado.

Las mediciones fueron realizadas en tres estructuras diferentes: aire, grasa y músculo. En todas las imágenes de nuestro estudio, se trazó un área constante para todas ellas, de $12 \mathrm{~mm}^{2}$, en cada una de las tres estructuras referidas, todas mediante planimetría manual, evaluando de forma automática la medida de la región de interés -ROI- en regiones que se correspondían con aire $(A)$, grasa $(G)$ o músculo $(M)$; como se aprecia en la Fig. 1. y se valoró su intensidad de señal, de acuerdo con la escala de gris para cada región empleando el programa OSIRIS; en resumen, en cada estructura se efectuó la medición en tres 
ocasiones y se obtuvo su intensidad de señal de forma automática; se calculó el promedio de las tres medidas realizadas y la media resultante se tomó como valor de referencia para dicha estructura.

Para las mediciones de las áreas empleamos el programa informático OSIRIS (versión 4.19), programa especialmente diseñado para la manipulación y análisis de imágenes médicas, desarrollado en el Hospital Cantonal Universitario de Ginebra. Dicho programa fue instalado en un ordenador personal con un procesador Intel ${ }^{\circledR}$ Centrino Duo ${ }^{\circledR}$ de 1,6 GHz, 2,00 Gb de memoria RAM y placa gráfica ATI Mobility Radeon x1300, de $128 \mathrm{Mb}$ de memoria.

Las mediciones se realizaron mediante planimetría manual (ratón óptico con resolución de movimiento de $800 \mathrm{dpi}$ ), usando las herramientas ofrecidas por el programa informático y obteniéndose los resultados de forma automática en lo que se refiere a las medidas de áreas y a los valores de intensidad de grises.

Fig. 1. VISUALIZACIÓN DEL TRAZO DE LA ROI (12 $\left.\mathrm{mm}^{2}\right)$ EN LAS DIFERENTES ESTRUCTURAS: AIRE (A), GRASA (G) Y MÚSCULO (M), EN UN CORTE CORONAL

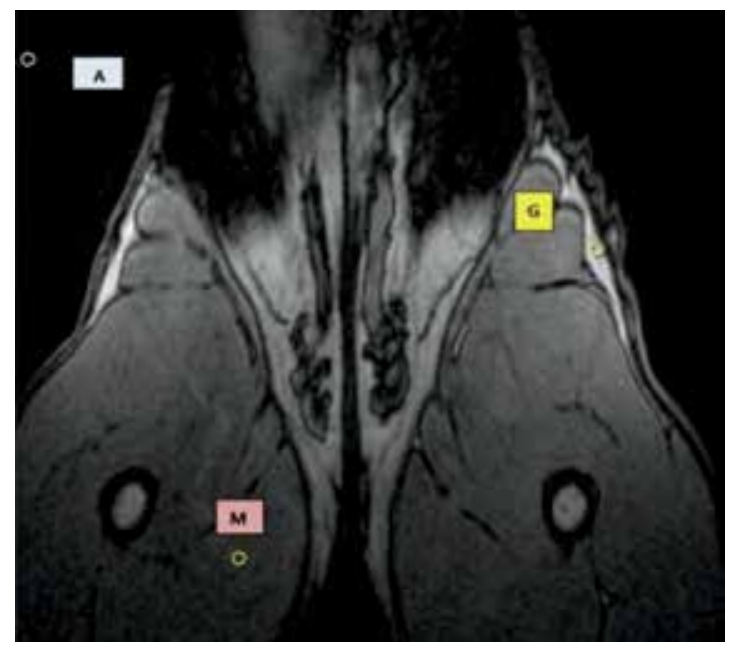

Fuente: Jaramillo y col, 2011

\subsection{Metodología y técnica empleada para establecer las características de intensidad de señal en la glándula mamaria de hembras sanas}

En el estudio de la mama de las hembras sanas empleamos series ponderadas en T1 por el mejor detalle anatómico que muestran. La glándula mamaria se estudió en cortes transversales, pues permiten diferenciar de forma segura y eficiente el tejido mamario como se visualiza en la Fig. 2, y, una vez se identificó claramente la glándula mamaria en todas las imágenes, se trazó en él la ROI de $20 \mathrm{~mm}^{2}$ tal como se ve en la Fig. 3.

Fig. 2. IMAGEN EN T1 DONDE SE APRECIA LA GLÁNDULA MAMARIA EN UN CORTE TRANSVERSAL $(\rightarrow)$

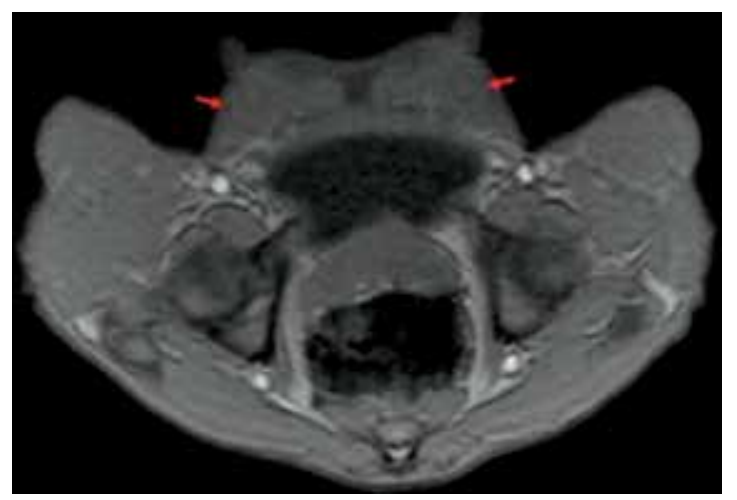

Fuente: Jaramillo y col, 2011.

Dicho procedimiento, en todos y cada uno de los casos, se realizó en tres ocasiones, con el fin de determinar un valor medio de la intensidad de señal de la glándula mamaria, definiendo así un valor de referencia del tejido glandular mamario y, de esta forma, identificar el tejido con una intensidad de señal propia.

Fig. 3. IMAGEN EN T1 DE UN CORTE TRANSVERSAL EN EL QUE SE OBSERVA LA DELIMITACIÓN DE UN ÁREA ESPECÍFICA $\left(20 \mathrm{~mm}^{2}\right)$ DENTRO DE LA MAMA SANA $(\rightarrow)$ Y LA OBTENCIÓN AUTOMÁTICA DEL VALOR DE SU INTENSIDAD DE SEÑAL

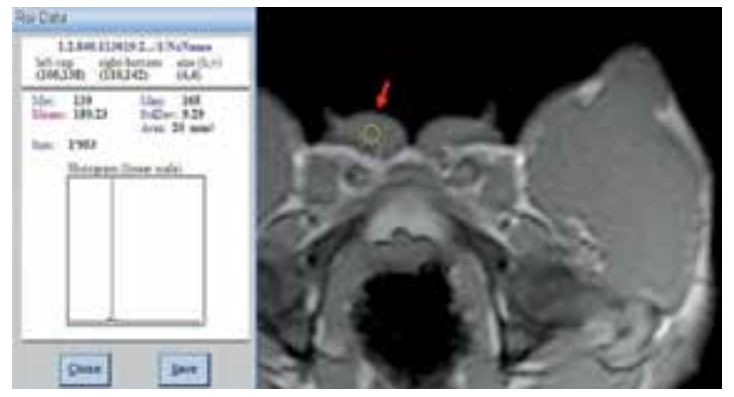

Fuente: Jaramillo y col, 2011.

\subsection{Tratamiento estadístico}

Los resultados de intensidad de señal de las diferentes estructuras estudiadas fueron expresados como media \pm desviación estándar. Para la comparación de estos valores empleamos la prueba ANOVA, proporcionando el valor del estadístico $(F)$ y su correspondiente $(p)$ se consideró que las 
diferencias eran estadísticamente significativas cuando $p$ fue menor a 0,05 (nivel de significancia del 95\%). Todo el análisis estadístico fue realizado con el programa Epi Info versión 6.04 [15].

\section{RESULTADOS Y DISCUSIÓN}

Validación de la técnica y metodología: el estudio comparativo de las intensidades de señal obtenidas en estas tres estructuras demostró la existencia de diferencias estadísticamente significativas entre ellas $(F=1857,1, p<0,001)$. Cuando comparamos el promedio de la intensidad de señal entre ellas, consideradas individualmente, la diferencia entre las intensidades de señal del aire y el músculo resultó significativa ( $F=30,7, p<0,001)$; de igual forma, cuando se comparó el promedio de la IS del aire con el promedio de IS de la grasa la diferencia fue estadísticamente significativa ( $F=34,6, p<0,001)$; del mismo modo, la diferencia entre el promedio de IS del músculo y de la grasa se mostró estadísticamente significativa $(F=23,2, p<0,001)$.

La Tabla I refleja el promedio de la intensidad de señal y la desviación estándar (DS) de las mediciones realizadas en las estructuras aire, grasa y músculo.

TABLA I

PROMEDIO DE LA INTENSIDAD DE SEÑAL DE LAS ESTRUCTURAS EN ESTUDIO EN LA VALIDACIÓN DE LA TÉCNICA

\begin{tabular}{|c|c|c|}
\hline & IS media & DS \\
\hline Aire & 5,2 & 1,2 \\
\hline Músculo & 84,9 & 12,1 \\
\hline Grasa & 276,9 & 36,8 \\
\hline
\end{tabular}

Fuente: autores

\subsection{Caracterización de la glándula mamaria en modelo animal canino mediante IRM, intensidad de señal (IS)}

La Fig. 3 muestra el corte transversal de la glándula mamaria de uno de los animales empleados en nuestro estudio, utilizado para valorar la intensidad de señal del tejido glandular mamario en hembras sin procesos patológicos en la glándula mamaria.

En cuanto al tipo de imagen y la dirección del corte, en nuestro estudio las imágenes ponderadas en T1 y en corte transversal permitieron, en el cien por cien de los casos, identificar la estructura glandular mamaria con mayor facilidad que cualquier otro corte empleado, habiendo logrado en todos los animales con las citadas imágenes pondera- das en T1, de los cortes transversales, diferenciar claramente el tejido glandular, el pezón y su separación con el músculo recto del abdomen como se observa en la Fig. 4.

Fig. 4. IMAGEN PONDERADA EN T1, EN CORTE TRANSVERSAL, DONDE SE APRECIAN LAS DOS GLÁNDULAS MAMARIAS. TEJIDO GLANDULAR MAMARIO (A). PEZÓN (B). SEPARACIÓN ENTRE EL TEJIDO GLANDULAR MAMARIO Y EL MÚSCULO RECTO ABDOMINAL (C). MÚSCULO RECTO ABDOMINAL (D).

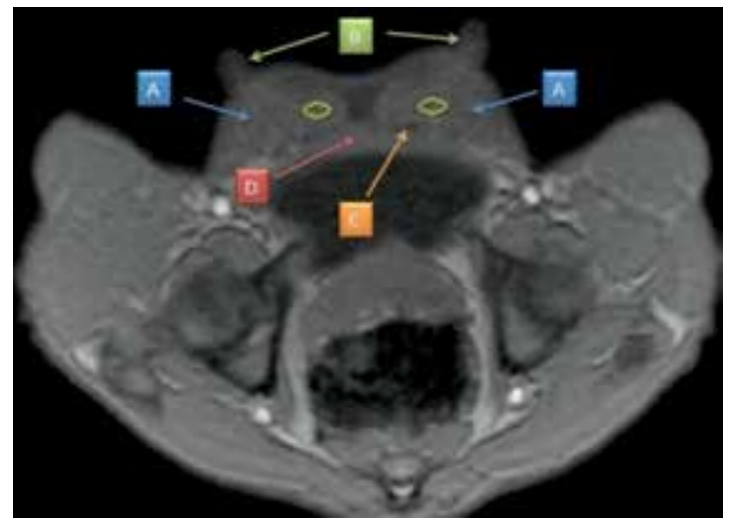

Fuente: Jaramillo y col, 2011.

En las imágenes ponderadas en T1, la estructura de la glándula mamaria se observó como una estructura homogénea e hiperintensa con relación al tejido muscular e hipointensa con relación a la grasa, pero claramente diferenciable del tejido muscular y demás tejidos circundantes, en el $100 \%$ de las imágenes obtenidas.

La intensidad de señal promedio de la glándula mamaria fue de $184,8 \pm 28,2$. Si consideramos separadamente las glándulas mamarias derecha e izquierda, las medias resultan prácticamente idénticas: $184,8 \pm 28,4$ en la mama derecha y de $184,8 \pm 28,1$ en la izquierda, sin diferencias estadísticamente significativas entre ellas, como se muestra en la Tabla II.

TABLA II

PROMEDIO DE INTENSIDAD DE SEÑAL EN LA GLÁNDULA MAMARIA SANA

\begin{tabular}{|c|c|c|c|}
\hline & $\begin{array}{c}\text { Glándula } \\
\text { mamaria }\end{array}$ & $\begin{array}{c}\text { Glándula } \\
\text { derecha }\end{array}$ & $\begin{array}{c}\text { Glándula } \\
\text { izquierda }\end{array}$ \\
\hline Promedio IS & 184,8 & 184,8 & 184,8 \\
\hline DS & 28,2 & 28,4 & 28,1 \\
\hline
\end{tabular}

Fuente: autores

\subsection{Intensidad de la señal relativa (IR) de la glándula mamaria de las hembras sanas}

Para determinar la IR de la glándula mamaria se tomó como referencia el promedio de intensidad de señal del aire, del músculo, de la grasa y de los ganglios de los animales en estudio (Tabla 
III), observándose que el promedio de la intensidad de la señal de la glándula mamaria resulta un $97,2 \%$ superior al promedio de intensidad de la señal del aire, siendo esta una diferencia estadísticamente significativa $(F=22,05, p=0,001)$, y un $51,3 \%$ mayor que el promedio de intensidad de la señal del músculo, también en este caso con significación estadística en esta diferencia ( $\mathrm{F}=$ $11,7, p=0,004)$. Por el contrario, el promedio de la intensidad de la señal de la mama es un 33,2\% menos intensa que el promedio de intensidad de la señal de la grasa, siendo asimismo esta diferencia estadísticamente significativa $(F=56,68$, $\mathrm{p}<0,001)$. Cuando comparamos el promedio de intensidad de señal de la glándula mamaria con el promedio de intensidad de señal del ganglio linfático encontramos que esta fue un $72,2 \%$ superior a la del ganglio, siendo la diferencia estadísticamente significativa $(F=16,39, p=0,002)$, como se puede apreciar en la Tabla III.

TABLA III

PROMEDIO DE INTENSIDAD DE SEÑAL DE LAS ESTRUCTURAS ESTUDIADAS

\begin{tabular}{|l|c|c|}
\hline Estructura & Promedio de intensidad (IS) & DS \\
\hline Aire & 5,2 & 1,2 \\
\hline Músculo & 84,9 & 12,1 \\
\hline Grasa & 276,9 & 36,8 \\
\hline Glándula mamaria & 184,8 & 28,2 \\
\hline Ganglio inguinal & 51,3 & 5,4 \\
\hline
\end{tabular}

Fuente: autores

\section{DISCUSIÓN}

Para llevar a cabo la validación de la técnica y de la metodología empleadas en lo referente a mediciones realizadas mediante técnica de planimetría manual, optamos por las IRM ponderadas en T1, al ser estas las que nos ofrecieron mayor calidad y definición para el trazado manual de las diferentes áreas por estudiar, considerando siempre la mejor visualización de la estructura en estudio por encima de la eficacia que en el diagnóstico clínico nos podía aportar la imagen, ya que, como se ha demostrado [16], [17], el manejo de las secuencias de pulsos hace prevalecer determinada información mediante la potenciación de la imagen. Coincidimos así con los autores de numerosos estudios que describen el empleo de técnicas de planimetría manual, utilizando el mismo tipo de imágenes, tanto en trabajos desarrollados con equipos de resonancia magnética de bajo campo (0,2 Teslas) [14], [18], [19], como es el caso de nuestro estudio, como en investigaciones llevadas a cabo con equipos de resonancia magnética de alto campo (1,5 a 2 Teslas) [20], [21], [22], [23], [24], [25].

La elección de las tres estructuras (aire, grasa y músculo) de las que se obtendrían, en nuestras imágenes por resonancia magnética ponderadas en T1, los valores medios de Intensidad de Señal que nos habrían de permitir crear un patrón de IS para cada estructura y diferenciarlas así de otras estructuras de interés en este estudio, se hizo de acuerdo con el hecho, reafirmado en numerosos trabajos de investigación [12], [13], [14], [17] de que en las imágenes ponderadas en T1 la grasa proporciona la máxima intensidad de señal (que se traduce en el blanco más intenso) y la señal que aporta el aire es la mínima (por lo que ofrece un color negro), mientras que la imagen del músculo resulta con una señal intermedia entre las dos anteriores, a consecuencia de la relación entre la densidad de los núcleos de hidrógeno de estas estructuras y el entorno bioquímico en el que se encuentran, así como de las secuencias de pulsos que tienen lugar en esta potenciación de la imagen, como se ha descrito anteriormente [16], [17].

La baja intensidad de la señal que obtuvimos al trazar una ROI de $12 \mathrm{~mm}^{2}$ en el área correspondiente al aire en todas la IRM del estudio (5,2 $\pm 1,2$ ) se explica así por la ausencia de tejido en esta zona, de modo que la baja densidad, e incluso ausencia prácticamente total de núcleos de hidrógeno, determina la drástica reducción de la intensidad de señal detectada al emplear la herramienta del programa informático mencionada para tal efecto, como queda también reflejado en las publicaciones revisadas en las que se afirma que, en las imágenes ponderadas en T1, el aire se verá más negro al determinar la menor intensidad de señal [17], [26], [27], [28].

En nuestro estudio, la IS de la grasa en las IRM de las hembras sin procesos morbosos en la glándula mamaria, medida de nuevo en un trazo de 12 $\mathrm{mm}^{2}$ sobre la estructura correspondiente, resultó ser de 276,9 $\pm 36,8$. Estos datos concuerdan con Io descrito por Gili [17], Dennis [26], Snellman [27] y Thrall [28], que reportan que la grasa en imágenes ponderadas en T1 se aprecia como una 
estructura brillante debido a su elevada intensidad de señal.

De igual forma, tras trazar una ROI de $12 \mathrm{~mm}^{2}$ para la determinación de las características del músculo en las IRM de nuestro estudio, obtuvimos una intensidad de señal de 84,9 $\pm 12,1$; valor que coincide con lo reportado por la bibliografía [17], [26], [27], [28], en trabajos que establecen que la intensidad de señal del músculo es intermedia, determinando en la imagen un tono que se sitúa en la mitad de una escala de grises.

Al realizar el estudio comparativo de los resultados de la valoración de la IS en estas tres estructuras, comprobamos que las diferencias entre ellas, tanto tomadas conjuntamente como comparadas de dos en dos, resultaron altamente significativas, coincidiendo con las afirmaciones de Gili [17], Dennis [18], Snellman [27] y Thrall [28], que definen la grasa como hiperintensa, el músculo como hipointenso y el aire negro en una imagen ponderada en T1, lo que refuerza nuestra decisión de tomar estos parámetros como patrón de comparación frente a las demás estructuras en estudio.

Caracterización de la glándula mamaria de la perra mediante IRM, intensidad de señal (IS): en nuestro estudio, las imágenes ponderadas en T1 sobre el plano transversal permitieron identificar la estructura mamaria con mayor facilidad y versatilidad que cualquier otro tipo de imagen o corte, en el cien por cien de los casos, ya que, como afirma Garamvölgyi [29], el uso de este tipo de plano y serie resalta las características anatómicas de estas estructuras, permitiendo, como fue nuestro caso, diferenciar claramente el tejido glandular, el pezón y el límite del tejido mamario con respecto al músculo recto abdominal.

Aun cuando no es nuestra intención, ni estamos en condiciones de dirimir la falta de consenso en la descripción de las IRM mamarias en la mujer, por parte de los autores de la bibliografía consultada, que describen numerosas características morfológicas y cinéticas para la caracterización del tejido mamario sano y afectado por lesiones benignas o malignas y mencionan dificultades para comparar las diferentes IRM mamarias incluso de un mismo paciente [30]-[38], en nuestro estudio hemos podido determinar las características generales del tejido glandular mamario como una estructura homogénea, que con una IS media de
$184,8 \pm 28,2$ resulta hiperintensa con relación al tejido muscular e hipointensa en relación al tejido adiposo, siendo las diferencias de IS con dichos tejidos estadísticamente significativas, por lo que sus características permiten claramente diferenciar en la IRM la mama de los tejidos circundantes, como se refleja en la Tabla III.

\section{CONCLUSIONES}

El empleo de un equipo de resonancia magnética de bajo campo proporciona suficiente información para el estudio de la glándula mamaria en la especie canina, así como de los ganglios linfáticos regionales, permitiendo la completa evaluación de su anatomía y la identificación precisa de cada estructura (tejido glandular, ganglio regional) merced a una Intensidad de Señal propia y característica para cada una siendo esta altamente eficaz en la determinación de intensidad de señal de cada estructura.

\section{AGRADECIMIENTOS}

El autor quiere expresar su agradecimiento a la doctora Alicia Ester Serantes Gómez por todo su apoyo e interés científico durante la realización de este trabajo, ya que sin su orientación y dedicación no hubiese culminado este estudio. De igual forma quiero agradecer a todo el equipo que me colaboró durante la realización de los protocolos de anestesia y adquisición de imágenes muy especialmente al doctor José Manuel Gonzalo Orden y a la doctora Marta Reguiero Purriños. Por último, y no menos importante, quiero expresar mi agradecimiento por todo su acompañamiento durante el desarrollo del estudio a los doctores Jesús Sánchez García, Alexandre Duarte, Francisco Jiménez, a la doctora Johanna Fonseca y a todos los que quisieron aportar durante el desarrollo de este estudio en la Unidad de Cirugía y Diagnóstico por Imagen de la Facultad de Veterinaria de la Universidad de León.

\section{REFERENCIAS}

[1] W.J. Banks, "Istoligia e anatomía microscopica veterinaria,” Ed. Piccin, (1991), pp. 365-369.

[2] T.E. Ayllon, A. Flores, A.J. Alés, "Caracterización de tumores mamarios en la perra". Curso Oncología Mamaria en la perra, Torremolinos (Málaga), (1996), http: // www. Veterinaria.org/ajfa/col I.htm.) 
[3] L. Marconato; F. Del Piero, "Oncologia medica dei piccoli animali," Poletto editore Srl., pp. 2-16, 47-50, 440459, 670-676, 679-681, (2005).

[4] S.H. Done, P.C. Goody, S.A. Evans, N.C. Stickland. "Atlas en color anatomía veterinaria. El perro y el gato," 1997, capítulo 8, p. 27.

[5] R. Barone, "Anatomia comparata dei mammiferei domestici," Splancnologia," Ed agricole, vol. 4, pp. 404406, (1994).

[6] C.R. Luiz, M.A. Miglino, T.C. Santos, “Segmentos anatomo-cirurgicos arteriais da glandula mamaria em caes," Archives of veterinary science, vol. 7, no. 1, pp. 27-36, (2002).

[7] A. Merighi, "Anatomia applicata e topografia regionale veterinaria," Ed. Piccin, (2005), pp. 113-152.

[8] G. Aguggini, V. Beghelli, L.F. Giulio, "Fisiologia degli animali domestici con elemti di etología," UTET, (2000), pp. 810-832.

[9] P.A. Fowler, C.E. Casey, G.G. Cameron, M. Foster, C. Knigth, "Cyclic changes in composition and volume of the breast during the menstrual cycle, measured by magnetic resonance imaging". Br J Obstetr Gynaecol, vol. 97 , no. 7 , pp. 595-602, (1990).

[10] A. Rieber, H.J. Brambs, V. Heilmann, R. Kreienberg, T. K" uhn, "Breast MRI for monitoring response of primary breast cancer to neo-adjuvant chemotherapy". Eur Radiol., vol. 12, no. 7, pp. 1711-9, (2002).

[11] C.K. Kuhl, R.K. Schmutzler, C.C. Leutner, "Breast MR imaging screening in 192 women proved or suspected to be carriers of a breast cáncer susceptibility gene: preliminary results". Radiology, vol. 215, no. 1, pp. 267-279, (2000).

[12] L. Millán, “Aplicación de la imagen por resonancia magnética al estudio de las patologías que afectan a la columna vertebral del perro". Tesis Doctoral. Facultad de Veterinaria. Universidad de León. León, España, (2000).

[13] R. Godoy, "Diagnóstico de tumores cerebrales primarios en caninos mediante imagen por resonancia magnética". Tesis Doctoral. Facultad de Veterinaria. Universidad de León. León, España, (2005).

[14] A.M. Duarte, "Estudio mediante resonancia magnética del cerebro del perro geriátrico". Tesis Doctoral. Facultad de Veterinaria. Universidad de León. León, España, (2008).

[15] A.G, Dean, A.J. Dean, D. Coulombier, A.H. Burton, K.A. Brendel, D.C. Smith, R.C. Dicker, K.M. Sullivan, R.F. Fagan, Epi Info, Version 6,04: a word processing, database, and statistics program or epidemiology on microcomputer, (1994).
[16] A. Desgrez, J. Bittoun, I. Idy-Peretti, "Bases físicas de la IRM," Cuadernos de IRM. Barcelona, España. Ed. Mason, S.A., (1991).

[17] J. Gili, "Introducción biofísica a la resonancia magnética," Barcelona, España. Ed. Centre Diagnóstic Pedralbes, (1993).

[18] S. Kii, Y. Uzuka, Y. Taura, M. Nakaichi, A. Takeuchi, H. Inokuma, T. Onishi, "Magnetic resonance imaging of the lateral ventricles in beagle-type dogs," Veterinary Radiology \& Ultrasound, vol. 38, no. 6, pp. 430-433, (1997).

[19] B.E. Ratsch, S. Kneissl, C. Gabler, "Comparative evaluation of the ventricles in the Yorkshire terrier and the German shepherd dog using low-field MRI," Veterinary Radiology \& Ultrasound, vol. 42, no. 5, pp. 410-413, (2001).

[20] T. Vullo, E. Korenman, R.P. Manzo, D.G. Gomez, M.D.F. Deck, P.T. Cahill, "Diagnosis of cerebral ventriculomegaly in normal adult Beagles using quantitative MRI," Veterinary Radiology \& Ultrasound, vol. 38, no. 4, pp. 277-281, (1997).

[21] M-Y. Su; E. Head, W.M. Brooks, Z. Wang, B. Muggenburg, G.E. Adam, R. Sutherland, C.W. Cotman, O. Nalcioglu, "Magnetic resonance imaging of anatomic and vascular characteristics in a canine model of human aging," Neurobiology of Aging, vol. 19, no. 5, pp. 479485, (1998).

[22] M-Y. Su, E. Head, B. Muggenburg, G.J-Y. Chiou, J. Wang, W.M. Brooks, R, Lee, C.W. Cotman, O. Nalcioglu, “MRI measurement of changes in the aging canine brain. Presented at the Symposium on Brain Aging and Related Behavioral Changes in Dogs: 13-16, (2002).

[23] V. Ho, S. Allen, M. Hood, "Renal Masses: Quantitative Assessment of Enhancement with Dynamic MR Imaging," Radiology, vol. 224, pp. 695-700, (2002).

[24] D. Tapp, C.T. Siwak, F.Q. Gao, J.Y. Chiou, S.E. Black, E. Head, B.A. Muggenburg, C.W. Cotman, N.W. Milgram, L.M. Su, "Frontal lobe volume, function, and $\beta$-amyloid pathology in a canine model of aging," Journal of Neuroscience, vol. 24, no. 38, pp. 8205-8213, (2004).

[25] P.D. Tapp, K. Head, E. Head, N.W. Milgram, B.A Muggenburg, M-Y. Su, "Application of an automated voxel-based morphometry technique to assess regional gray and white matter brain atrophy in a canine model of aging," Neuroimage, vol. 29, pp. 234-244, (2006).

[26] R. Dennis, An introduction to veterinary CT and MR scanning. Vet. Annual, vol. 36, pp. 17-40, (1996).

[27] M. Snellman, "Magnetic resonance imaging in canine spontaneous neurological disorders: an evaluation of equipment and methods". Academic dissertation. University of Helsinki, Finland, (2000), pp. 32-64, 
[28] D. Thrall, “Principios físicos de la tomografía computarizado y de la resonancia magnética," Manual de Diagnóstico radiológico veterinario, Cuarta edición. Clifford R. Berry. (2003), Capítulo 3.

[29] R. Garamvölgyi, Z. Petrási, A. Hevesi, C. Jakab, Z. Vajda, P. Bogner, I. Repa, "Magnetic resonance imaging technique for the examination of canine mammary tumours," Acta Vet Hung., Jun; vol. 54, no. 2, 143-59, (2006).

[30] L.W. Nunes, M.D. Schnall, S.G. Orel, M.G. Hochman, C.P. Langlotz, C.A. Reynolds, M.H. Torisian, "Breast MR imaging: interpretation model,” Radiology. vol. 202, no. 3, pp. 833-41, (1997).

[31] L.W. Nunes, M.D. Schnall, E.S. Siegelman, C.P. Lanflotz, S.G. Orel, D. Sullivan, L.A. Muenz, C.A. Reynolds, M. H. Torosian, "Diagnostic performance characteristics of architectural features revealed by high spatialresolution MR imaging of the breast," Am J Roentgenol, vol. 169, pp. 409-15, (1997).

[32] K. Kinkel, T.H. Helbich, L.J. Esserman, “Dynamic highspatial-resolution MR imaging of suspicious breast lesions: diagnostic criteria and interobserver variability," Am J Roentgenol, vol. 175, no. 1, pp. 35-43, (2000).

[33] M.D. Schnall, S. Rosten, S. Englander, S.G. Orel, L.W. Nunes, "A combined architectural and kinetic interpretation model for breast MR images," Acad Radiol., vol. 8, pp. 591-7, (2001).
[34] L.W. Nunes, M.D. Schnall, S.G. Orel, "Update of breast MR imaging architectural interpretation model," Radiology, vol. 219, no. 2, pp. 484-94, (2001).

[35] L. Liberman, E.A. Morris, M.J. Lee, J. Kaplan, L. La Trenta, J. Menell, A. Abramson, S. Dashnaw, D. Ballon, D. Dershaw, "Breast lesions detected on MR imaging: features and positive predictive value," Am J Roentgenol., vol.179, pp. 171-8, (2002)..

[36] L. Liberman, E.A. Morris, D.D. Dershaw, A.F. Abramson, Tan, L.K., "Ductal enhancement on MR imaging of the breast," Am J Roentgenol, vol. 181, pp. 519-25, (2003).

[37] M.D. Schnall, J. Blume, D.A. Bluemke, G. DeAnglelis, N. DeBruhl, S. Harms, S. Heywang-Köbrunneret, N. Hylton, Ch. Kuhl, E Pisano, P. Causer, S. Schnitt, D Thickman, S. Stelling, P. Weatherall, C. Lehman, C. Gatsonis, "Diagnostic architectural and dynamic features at breast MR imaging: multicenter study," Radiology, vol. 238, pp. 42-53, (2006).

[38] A. Tardivon, A. Athanasiou, F. Thibault, C. El khoury, "Breast imaging and reporting data system (BIRADS): Magnetic resonance imaging," European Journal of Radiology, vol. 61, pp. 212-215, 2007. 